\title{
Risk Stratification prior to Cardiac Surgery: Another Important Lesson Learned
}

\author{
John L. Jefferies \\ University of Tennessee Health Science Center, Memphis, TN, USA
}

\begin{abstract}
"Measure what can be measured, and make measurable what cannot be measured." - Galileo.

In this issue of the journal, Amatruda and colleagues [1] report data from their prospective study assessing the utility of pre- and postoperative urine alpha-1-microglobuin (Ua1m) levels to predict adverse outcomes following cardiac surgery. In brief, this prospective multicenter analysis performed by the Translational Research Investigating Biomarker Endpoints for Acute Kidney Injury (TRIBE-AKI) assessed outcomes and a robust cohort of nearly 1,500 adults. The predefined outcomes included postoperative acute kidney injury (AKI) during index hospitalization and longitudinal risks for chronic kidney disease (CKD) incidence and progression, cardiovascular (CV) events, and all-cause mortality after discharge.

There were $230 \mathrm{AKI}$ events during the time of cardiac surgery hospitalization. During a median follow-up of 6.7 years, there were 212 cases of incident CKD, 54 cases of CKD progression, 269 CV events, and 459 deaths. Preoperative Ualm was independently associated with the development of a KI, CKD progression, and all-cause mortality. Interestingly, postoperative Ua1m levels provided no predictive power.

Too frequently in medicine, we are reactive as practitioners. We observe disease developing or worsening secondary to a lack of adequate predictive tools. Furthermore, there may be testing that can be pursued for risk
\end{abstract}

stratification. However, these tests may be inaccessible to the majority of requesting providers or deemed cost ineffective by payors. And, in the setting of planned cardiac surgery, patients may be critically ill or considered too unstable thereby limiting the opportunity to pursue comprehensive risk analysis. The need for testing that is userfriendly and easily added to existing care pathways is persistently apparent in nephrology and CV medicine. If a simple tool purely dependent on a urine sample were available to health professionals that provided a stratification mechanism in cardiac surgery patients, it could inform a thoughtful and aggressive monitoring strategy for events that negatively impact morbidity and mortality.

All patients undergoing elective cardiac surgery have a preoperative risk assessment. This is considered standard of care. Although each institution may have a preference for which scoring system used, the output from these calculations has significant implications. The score for an individual patient may be the deciding factor between pursuing surgery or denying surgery as an option secondary to deemed unacceptable risk. The Society of Thoracic Surgeons (STS) has developed a Short-Term Risk Calculator with the goal of predicting individual morbidity and mortality for the most commonly performed cardiac surgeries (https://www.sts.org/resources/risk-calculator) $[2,3]$. The score is calculated on clinical data such as planned surgery, gender, race, left ventricular ejection fraction, and a myri- 
ad of other determinants. The "last" serum creatinine is also included in this calculation. Given the possible impact of existing comorbidities such as diabetes or hypertension and the prevalence of the cardiorenal syndrome in these cardiac populations, relying on the most recent creatine level may be suboptimal. The European system for cardiac operative risk evaluation (EuroSCORE) has also been developed for risk calculation (http://www.euroscore.org/ calc.html) [4]. This score has been updated with the EuroSCORE II risk calculator [5]. The EuroSCORE II incorporates similar data to the STS score such as age, gender, left ventricular function, and type of cardiac surgery. However, the EuroSCORE II leverages creatinine clearance as calculated by the Cockroft-Gault equation instead of most recent creatinine. Both these scoring systems have been used for many years and validated across multiple populations. Notably, they have obvious inherent limitations with regard to assessing risk of AKI and development and/ or progression of CKD. As the authors appropriately note, creatinine reflects glomerular clearance but does not provide direct information about tubule injury or dysfunction. Isolated serum creatinine levels are not ideal for recognizing AKI, especially those secondary to kidney tubule damage. By extension of that knowledge, the use of creatinine to diagnose AKI limits opportunities to reliably identify future risk or progression of CKD.

The data presented by Amatruda and colleagues [1] may offer the opportunity to revisit these calculations. Ua1m is a marker of proximal tubule dysfunction and provides more timely indication of AKI [6]. As providers caring for adult patients undergoing cardiac surgery, we would benefit from any improvements that can be implemented in our traditional preoperative risk stratification strategies. The downstream implications of AKI are important and potentially catastrophic including future CV events. The cardiac populations that we are discussing would benefit from decreasing future risk whenever possible. The diagnosis of AKI has become a consistent benchmark of outcomes following procedures in both the catheterization laboratory and operating room. However, the avoidance of AKI continues to be a significant hurdle in healthcare secondary to limited opportunities for diagnosis and even less opportunities for treatment once diagnosed. The current report provides perplexing data that may afford us the unique opportunity to enhance avoidance strategies in patients that require intervention. They found that higher preoperative Ualm level was significantly associated with increased risk of HAI during index hospitalization, CKD progression, and all-cause mortality. Paradoxically, postoperative Ualm levels and the change between pre-and postoperative Ua1m levels were not strongly associated with outcomes following discharge. As opposed to waiting on postoperative creatinine levels which lag by definition, we now have the opportunity to identify patients with $36 \%$ higher odds of developing AKI in the setting of an elevated preoperative Ualm level.

Major transformations have occurred over the past few years in CV intervention. No longer is it absolutely required to undergo coronary artery bypass grafting, aortic valve replacement, and mitral valve repair via traditional surgical approaches. We now can effectively deliver these interventions percutaneously via complex coronary interventions, transcatheter aortic valve replacement, and transcatheter mitral valve repair $[7,8]$. Importantly, the models mentioned above were not developed with consideration of these new procedures. They have not been broadly validated for these rapidly expanding technologies. As such, the use of novel biomarkers such as Ualm should be strongly considered as we revisit the changing landscape of CV interventions. These new techniques offer interventions to populations that in the past would have been denied surgery. An understanding of the true risks associated with these procedures is paramount to ensuring good outcomes given the underpinnings of larger heterogeneity, higher degree of risk, and required exposure to contrast agents in the catheterization laboratory.

The compelling findings reported by Amatruda and colleagues [1] may inform strategies which reconfigure our planning strategies for patients undergoing elective cardiac surgery. Armed with a better tool which can identify AKI prior to surgery, we can more effectively stratify patients. This could be a powerful opportunity in the preoperative planning of these patients including avoidance of nephrotoxic agents and optimizing medical therapies such as those used in the treatment of heart failure. As a community, we recognize that AKI will still occur in some patients regardless of our best efforts. But, we would be remiss if we did not exercise every opportunity to identify high-risk candidates. By increasing awareness of these patient cohorts, we can provide a more thoughtful preoperative approach and plan for postoperative management. This offers points of intervention which may impact cost, length of stay, morbidity, and mortality. The findings presented need to be further validated and studied in a more rigorous manner. But, reported information is encouraging. If we could better understand postoperative outcomes by a simple preoperative urine test, why would we not do it? 


\section{Conflict of Interest Statement}

The author has no conflicts of interest to disclose.

\section{Funding Sources}

The author did not receive any funding.

\section{References}

1 Amatruda JGEM, Estrella MM, Garg AX, Thiessen-Philbrook H, McArthur E, Coca SG, et al. Urine alpha-1-microglobulin levels and acute kidney injury, mortality and cardiovascular events following cardiac surgery. Am J Nephrol. 2021. DOI: 10.1159/000518240.

2 Shahian DM, Jacobs JP, Badhwar V, Kurlansky PA, Furnary AP, Cleveland JC, et al. The society of thoracic surgeons 2018 adult cardiac surgery risk models: part 1-background, design considerations, and model development. Ann Thorac Surg. 2018;105:1411-8.

3 O’Brien SM, Feng L, He X, Xian Y, Jacobs JP, Badhwar V, et al. The society of thoracic surgeons 2018 adult cardiac surgery risk models: part 2-statistical methods and results. Ann Thorac Surg. 2018;105:1419-28.

4 Nashef SA, Roques F, Michel P, Gauducheau E, Lemeshow S, Salamon R. European system for cardiac operative risk evaluation (EuroSCORE). Eur J Cardiothorac Surg. 1999;16:913.

5 Nashef SA, Roques F, Sharples LD, Nilsson J, Smith C, Goldstone AR, et al. EuroSCORE II. Eur J Cardiothorac Surg. 2012;41:734-5. Discussion 744-5.

6 Heise D, Rentsch K, Braeuer A, Friedrich M, Quintel M. Comparison of urinary neutrophil glucosaminidase-associated lipocalin, cystatin C, and $\alpha 1$-microglobulin for early detec- tion of acute renal injury after cardiac surgery. Eur J Cardiothorac Surg. 2011;39:38-43.

7 Ono M, Serruys PW, Hara H, Kawashima H, Gao C, Wang R, et al. 10-Year follow-up after revascularization in elderly patients with complex coronary artery disease. J Am Coll Cardiol. 2021;77(22):2761-73.

8 Writing Committee Members; Otto CM, Nishimura RA, Bonow RO, Carabello BA, Erwin JP, Gentile F, et al. 2020 ACC/AHA guideline for the management of patients with valvular heart disease: a report of the American College of Cardiology/American Heart Association joint committee on clinical practice guidelines. J Am Coll Cardiol. 2021;77(4):e25-197. 